\title{
A New Operational Matrix of Derivative for Orthonormal Bernstein Polynomial's
}

\author{
MayadaN.Mohammed Ali* \\ Received 16, May, 2013 \\ Accepted 26, September, 2013
}

\begin{abstract}
:
In this paper, an orthonormal family has been constracted $\left\{b_{i 6}\right\}_{i=0}^{6}$ of polynomials of degree six is first constructed by using Gram-Schmidt orthonormalization process on Bernstein polynomials $\left\{B_{i 6}\right\}_{i=0}^{6}$. Then, an orthonormal Bernstein operational matrix of derivative $D_{b}$ is derived.Finally, the orthonormal Bernstein expansions along with operational matrix of derivative are applied for variational problemsapproximately.
\end{abstract}

Keywords: Gram-Schmidt using direct method process, operational matrix of derivative, Bernstein polynomials, variational problems

\section{Introduction:}

Bernstein polynomials have been recently used for the solution of many problems [1-5].

In this paper, an approximate solution has been proposed to solve variationalproblems, using new operational matrix of derivative for orthonormal Bernstein function. Our matrix of derivative in order to reduce the problem into solving quadratic programming problem. The operational matrix of derivative $D_{b}$ is given by

approach is based on using operational

$$
\frac{d b(x)}{d x}=D_{b} B(x)
$$

where

$$
b(x)=\left[b_{06}(x), b_{16}(x), b_{26}(x), b_{36}(x), b_{46}(x), b_{56}(x), b_{66}(x)\right]
$$

$\operatorname{and} B_{i, 6}, i=0,1,2,3,4,5,6$ are basis Bernstein polynomials.Several papers have appeared in the literature concerned with the application of operational matrix of derivatives.Hosseini[6], applied the operational matrix of derivative for Chebyshev wavelets for solving ordinary differential equation with non analyticsolution, Doha[7] used the shifted Chebyshev operational matrix of fractional derivatives together with spectral method for solving fractional differential equations, while the operational matrix of derivative of five order orthonormal Bernstein polynomials was constructed and applied in solving variational problems[8].

\section{Definition of Bernstein Polynomials}

The Bernstein basis polynomials of degree $\mathrm{n}$ are defined on the interval $[0$, 1] as follows

$$
\begin{gathered}
B_{i, n}(x)=\left(\begin{array}{c}
n \\
i
\end{array}\right) x^{i}(1-x)^{n-i},(i \\
=0,1,2, \ldots n) \ldots(1) \\
\operatorname{set}\left\{B_{0, n}(x), B_{1, n}(x), \ldots, B_{n, n}(x)\right\} \quad \text { in }
\end{gathered}
$$
Hilbert space $L^{2}[0,1]$ is a complete basis.Therefore, any polynomial of degree $\mathrm{n}$ can be expanded in terms of linear combination of $B_{i, n}(x), i=$ $0,1, \ldots, n$, as

*University of Technology/Applied Science Department 


$$
f(x)=\sum_{i=0}^{n} c_{i} B_{i, n}(x)
$$

Define

$\Psi_{n}(x)=\left[B_{0, n}(x), B_{1, n}(x), \ldots, B_{n, n}(x)\right]$

Therefore, we can write [2]

where

$$
\Psi_{n}(x)=A T_{n}(x) \ldots(3)
$$

$$
T_{n}(x)=\left[1, x, x^{2}, \ldots, x^{n}\right]^{T}
$$

and $A_{i+1, j+1}=$

$\left\{\begin{array}{cl}(-1)^{j-i}\left(\begin{array}{c}n \\ i\end{array}\right)\left(\begin{array}{c}n-i \\ j-i\end{array}\right), & i \leq j \\ 0 & , i>j\end{array} i=\right.$

$0,1, \ldots, n$

The matrix $\mathrm{A}$ is invertible since $|A|=$ $\pi_{i=0}^{n}\left(\begin{array}{c}n \\ i\end{array}\right) \neq 0$.

\section{Obtaining Orthonormal \\ Bernstein Functions of Order \\ Six}

Different methods may be used to obtain orthogonal polynomials, namely, mostcommonly, the GramSchmidt method.

Using orthonormalization process on $\left\{B_{i, 6}\right\}_{i=0}^{6}$, a class of orthonormal polynomials can be obtained from Bernstein polynomials, denoted by $b_{06}, b_{16}, b_{26}, b_{36}, b_{46}, b_{56}, b_{66}$, and they are given by :

$$
\begin{aligned}
& b_{06}(x)=\sqrt{13}(1-x)^{6} \\
& b_{16}(x)=\sqrt{44}\left[6 x(1-x)^{5}\right.\left.-\frac{1}{2}(1-x)^{6}\right] \\
& b_{26}=11\left[15 x^{2}(1-x)^{4}\right. \\
& \quad-6 x(1-x)^{5} \\
&\left.+\frac{3}{11}(1-x)^{6}\right]
\end{aligned}
$$

$$
\begin{aligned}
& b_{36}=\sqrt{252}\left[20 x^{3}(1-x)^{3}\right. \\
& -\frac{45}{2} x^{2}(1-x)^{4} \\
& +5 x(1-x)^{5} \\
& \left.-\frac{11}{66}(1-x)^{6}\right] \\
& b_{46}=\frac{42}{\sqrt{5}}\left[15 x^{4}(1-x)^{2}-\right. \\
& 40 x^{3}(1-x)^{3}+\frac{180}{7} x^{2}(1-x)^{4}- \\
& \left.\frac{30}{7} x(1-x)^{5}+\quad \frac{5}{42}(1-x)^{6}\right] \\
& b_{56}=\frac{28}{\sqrt{3}}\left[6 x^{5}(1-x)\right. \\
& -\frac{75}{2} x^{4}(1-x)^{2} \\
& +60 x^{3}(1-x)^{3} \\
& -30 x^{2}(1-x)^{4} \\
& +\frac{30}{7} x(1-x)^{5} \\
& \left.-\frac{3}{28}(1-x)^{6}\right] \\
& b_{66}=7\left[x^{6}-18 x^{5}(1-x)\right. \\
& +75 x^{4}(1-x)^{2} \\
& -100 x^{3}(1-x)^{3} \\
& +45 x^{2}(1-x)^{4} \\
& -6 x(1-x)^{5} \\
& \left.+\frac{1}{7}(1-x)^{6}\right]
\end{aligned}
$$

Now, define

$$
\begin{aligned}
& b(x)=\left[b_{06}(x), b_{16}(x), b_{26}(x),\right. \\
& \left.b_{36}(x), b_{46}(x), b_{56}(x), b_{66}(x)\right]^{T} \text {, and } \\
& B(x)=\left[B_{06}(x), B_{16}(x), B_{26}(x), B_{36}(x),\right. \\
& \left.B_{06}(x), B_{06}(x), B_{66}(x)\right]
\end{aligned}
$$$$
\text { Therefore we can obtain }
$$$$
b(x)=A B(x)
$$

where

$\left[\begin{array}{ccccccc}3.605551 & 0 & 0 & 0 & 0 & 0 & 0 \\ -3.316625 & 6.633250 & 0 & 0 & 0 & 0 & 0 \\ 3 & -11 & 11 & 0 & 0 & 0 & 0 \\ -2.645751 & 13.228757 & -23.811762 & 15.874508 & 0 & 0 & 0 \\ 2.236068 & -13.416408 & 32.199379 & -37.565942 & 18.782971 & 0 & 0 \\ -1.732051 & 11.547005 & -32.331615 & 48.497423 & -40.414519 & 16.165808 & 0 \\ 1 & -7 & 21 & -35 & 35 & -21 & 7\end{array}\right]$

Matrix A is a $(7 \times 7)$ upper triangular matrix. 
The Operational Matrix of Derivative for Orthonormal Bernstein of Order Six

A function $f \in L^{2}[0,1]$ may be written as

$f(x)=\lim _{n \rightarrow \infty} \sum_{i=0}^{n} c_{\text {in }} b_{\text {in }}(x) \ldots$

where $c_{i n}=\left\langle f, b_{i n}\right\rangle$ and $\langle$,$\rangle is inner$ product on $L^{2}[0,1]$.If the series approximation $f^{*}$ of $f$ as

$f \cong f^{*}=\sum_{i=0}^{n} c_{i n} b_{i n}=c^{T} b(x) \ldots$

where, $\mathrm{c}=\left[c_{0 n}, c_{1 n}, \ldots, c_{n n}\right]^{T}$

and $b(x)=$

$\left[b_{0 n}(x), b_{1 n}(x), \ldots, b_{n n}(x)\right]^{T}$

Now, consider the case when $=6$, then eq. (6) becomes

$f=\sum_{i=0}^{6} c_{i 6} b_{i 6}=c^{T} b(x) \ldots$

where

$$
D_{b}=\left[\begin{array}{ccccccc}
-21.6333 & -3.6056 & 0 & 0 & 0 & 0 & 0 \\
59.6992 & -23.2164 & -13.2665 & 0 & 0 & 0 & 0 \\
-84 & 96 & 0 & -3 & 0 & 0 & 0 \\
95.2470 & -169.3281 & 84.6640 & 71.4353 & -63.4980 & 0 & 0 \\
-93.9149 & 212.4265 & -150.2638 & -40.2492 & 187.8297 & -93.9149 & 0 \\
79.6743 & -206.1140 & 235.5589 & -24.2487 & -242.4871 & 266.7358 & -96.9948 \\
-48 & 132 & -168 & 42 & 168 & -252 & 168
\end{array}\right]
$$

The matrix $D_{b}$ is a $(7 \times 7)$ matrix and it is called Bernstein Orthonormal polynomials of derivative.

Solution of Variational Problem

In this section, variational problem is solved by using the operational matrix of derivative for orthonormal Bernstein polynomials.
Here $c=\left[c_{06}, c_{16}, \ldots, c_{66}\right]^{T}$ and $b(x)=$ $\left[b_{06}(x), b_{16}(x), b_{26}(x), \ldots, b_{66}(x)\right]^{T}$ Differentiate eq. (7) with respect to $x_{n}$, to get :

$\dot{f}=c^{T} \dot{b}(x) \ldots(8)$

where

$c=\left[c_{06}, c_{16}, \ldots, c_{66}\right]^{T}$

and

$\dot{b}(x)=\left[\dot{b}_{06}(x), \dot{b}_{16}(x), \ldots, \dot{b}_{66}(x)\right]^{T}$

By substituting eq. (4) into (8), one can obtain

$$
\dot{f}=c^{T} A \dot{B}(x) \ldots(9)
$$

Since the derivative formula of $\dot{B}(x)$ is given by

$$
\begin{aligned}
\dot{B}_{k, n}(x)=(n & -k+1) B_{k-1, n}(x) \\
& -(n-2 k) B_{k, n}(x) \\
& -(k \\
& +1) B_{k+1, n}(x) \ldots(10)
\end{aligned}
$$

Therefore eq. (9) becomes $\dot{B}=c^{T} D_{b} B(x) \ldots(11)$
Consider the first order functional extremal with two fixed boundary conditions [9]

$$
J=\int_{0}^{1}\left[\dot{x}^{2}(t)+t \dot{x}(t)\right.
$$

with the following boundary conditions:

$$
x(0)=0, \quad x(1)=\frac{1}{4} \ldots
$$

The exact solution of this problem is$$
\left.+x^{2}(t)\right] d t \ldots(12)
$$ 
$x(t)$

$=\frac{-e^{-t}\left[\left(-1+e^{t}\right)\left(e-2 e^{2}-2 e^{t}+e^{1+t}\right)\right]}{4\left(-1+e^{2}\right)} \ldots$

At first, the function $x(t)$ is approximated by

$$
x(t)=c^{T} b(t) \ldots(15)
$$

where $c=\left[c_{06}, c_{16}, c_{26}, c_{36}, c_{46}\right.$,

$\left.c_{56}, c_{66}\right]^{T}$ is to be determined

Differentiated eq. (15), yields

$$
\dot{x}(t)=c^{T} \dot{b}(t)
$$

or $\dot{x}(t)=c^{T} D_{b} b(t) \ldots(16)$

substituting (15) and (16) into (12) to get

$$
\begin{array}{r}
J(x)=\int_{0}^{1}\left[c^{T} \dot{b}(t) \dot{b}^{T}(t) c+c^{T} t \dot{b}(t)\right. \\
\left.+c^{T} b(t) b^{T}(t) c\right] d t
\end{array}
$$

Let $H=2 \int_{0}^{1}\left[b^{T}(t) \dot{b}^{T}(t)+\right.$

$\left.b(t) b^{T}(t)\right] d t \ldots(17)$

$d^{T}=\int_{0}^{1} t \dot{b}^{T}(t) d t$

Then eq. (17) can be simplified to

$J(x)=\frac{1}{2} c^{T} H c+d^{T} c \ldots$

eq. (15) and the boundary conditions in (13) , imply $x(0)=c^{T} b(0)=0 \quad$ and $x(1)=$ $c^{T} b(1)=\frac{1}{4} \ldots$ (19)

The quadratic programming problem in eqs. (18) and (19) can be rewritten as follows

\begin{tabular}{|c|c|c|c|c|c|c|c|}
\hline & [ 6.6993 & -3.1958 & -1.7832 & -0.8951 & -0.3849 & -0.1287 & -0.0258 \\
\hline & -3.1958 & 3.7203 & 0.9720 & -0.2224 & -0.5020 & -0.3576 & -0.1287 \\
\hline & -1.7832 & 0.9720 & 1.3686 & 0.7076 & -0.0924 & -0.5020 & -0.3849 \\
\hline $\mathrm{H}=$ & -0.8951 & -0.2224 & 0.7076 & 1.1056 & 0.7076 & -0.2224 & $-0 . \varepsilon$ \\
\hline & -0.3849 & -0.5020 & -0.0924 & 0.7076 & 1.3686 & 0.9720 & -1.7832 \\
\hline & -0.1287 & -0.3576 & -0.5020 & -0.2224 & 0.9720 & 3.7203 & -3.1958 \\
\hline & $\mathrm{L}-0.0258$ & -0.1287 & -0.3849 & -0.8951 & -1.7832 & -3.1958 & 6.6993 \\
\hline & $=\left[\begin{array}{l}b^{T}(0) \\
b^{T}(1)\end{array}\right]$ & $=\left[\begin{array}{ll}1 & 0 \\
0 & 0\end{array}\right.$ & $\begin{array}{lll}0 & 0 & 0 \\
0 & 0 & 0\end{array}$ & $\left.\begin{array}{ll}0 & 0 \\
0 & 1\end{array}\right], d^{T}=$ & & $1-1$ & (5) \\
\hline
\end{tabular}

$\operatorname{minimize} J(x)=\frac{1}{2} c^{T} H c+d^{T} c$

subject to $F_{1} c-b_{1}=0$

where

The optimal values of unknown parameters $c^{*}$ can be obtained using Lagrange multiplier technique as

$$
\begin{aligned}
& c^{*} \\
& =-H^{-1} c \\
& +H^{-1} F_{1}^{T}\left(F_{1} H^{-1} F_{1}^{T}\right)^{-1}\left(F_{1} H^{-1} c\right. \\
& \left.+b_{1}\right),
\end{aligned}
$$

Here $\quad c^{*}=[0,0.0740,0.1313$, $0.1756,0.2093,0.2338,0.25]^{T}$

The optimal parameters $c^{*}$ are substituted in eq.(15) to obtain $x(t)$. In table (1), a comparison is made between the approximate values of $x(t)$ using the operational matrix of derivative of orthonormal Bernstein polynomials and Legenderwavelete method [10] with the exact solution.

Table (1) estimated and exact values of $x(t)$

\begin{tabular}{|c|c|c|c|}
\hline $\mathbf{t}$ & $\begin{array}{c}\text { LegenderWavelete } \\
{[\mathbf{1 2}]}\end{array}$ & $\begin{array}{c}\text { Present } \\
\text { method }\end{array}$ & exact \\
\hline 0.1 & 0.041949 & 0.04195073 & 0.04195073 \\
0.2 & 0.079315 & 0.07931715 & 0.07931715 \\
0.3 & 0.112471 & 0.11247322 & 0.11247322 \\
0.4 & 0.141749 & 0.14175080 & 0.14175080 \\
0.5 & 0.167443 & 0.16744292 & 0.16744292 \\
0.6 & 0.189807 & 0.18980669 & 0.18980669 \\
0.7 & 0.209064 & 0.20906593 & 0.20906593 \\
0.8 & 0.225411 & 0.22541340 & 0.22541340 \\
0.9 & 0.239010 & 0.23901272 & 0.23901272 \\
1 & 0.249999 & 0.25000000 & 0.25000000 \\
\hline
\end{tabular}

\section{The Convergence Test}

In the presented method, the states are expanded in terms of orthonormal Bernstein polynomials of order six,

$x_{i N}(t)=\sum_{k=1}^{N} a_{i k} b_{k}(t)$

Sothat

$$
\begin{aligned}
& x_{i}(t)=x_{i N}(t)+\sum_{k=N+1}^{\infty} a_{i k} b_{k}(t) \\
& \text { or } x_{i}(t)=x_{i N}(t)+r_{i}(t) \ldots(20)
\end{aligned}
$$


we must select coefficients in eq.(20) such that the norm of the residual function $\|r(t)\|$ is less than some convergence criterion $\varepsilon$, where $r(t)=\max \left(r_{1}(t), r_{2}(t), \ldots, r_{N}(t)\right) \quad$. The most useful test of convergence in terms of $\mathrm{N}$ comes from examining the $L^{2}$ norm of $x_{i}, i=1,2, \ldots n$ (the state variables that is approximated), that is $\left[\int_{0}^{1}\left(x_{i}(t)-x_{i N}(t)\right)^{2} d t\right]^{1 / 2}<$ $\varepsilon_{i}, \quad i=1,2, \ldots, n$

Let $\varepsilon=\max \left(\varepsilon_{1}, \varepsilon_{2}, \ldots, \varepsilon_{n}\right)$, therefore $\left[\int_{0}^{1}\left(x(t)-x_{N}(t)\right)^{2} d t\right]^{1 / 2}<\varepsilon$ Hence

$$
\begin{gathered}
{\left[\int _ { 0 } ^ { 1 } \left(\sum_{i=0}^{N+M} a_{i} b_{i}(t)\right.\right.} \\
\left.\left.-\sum_{i=0}^{N} a_{i} b_{i}(t)\right)^{2} d t\right]^{1 / 2} \\
<\varepsilon
\end{gathered}
$$

Where $M \geq 1$

$$
\begin{aligned}
& {\left[\int_{0}^{1}\left(\sum_{i=N+1}^{N+M} a_{i} b_{i}(t)\right)^{2} d t\right]^{1 / 2}<\varepsilon} \\
& {\left[\int_{0}^{1}\left(\sum_{i=N+1}^{N+M} a_{i} b_{i}(t)\right)\right.} \\
& \left.-\left(\sum_{i=N+1}^{N+M} a_{i} b_{i}(t)\right) d t\right]^{1 / 2}<\varepsilon \\
& \sum_{i=N+1}^{N+M} \sum_{j=N+1}^{N+M} a_{i} a_{j} \int_{0}^{1} b_{i}(t) b_{j}(t) d t<\varepsilon
\end{aligned}
$$

Since the Bernstein polynomials of order six $b_{i}(t)$ is orthonormal, therefore eq. (21) reduces to the simple form

$$
\sum_{i=N+1}^{N+M} a_{i}^{2}<\varepsilon .
$$

\section{Conclusion:}

The seven polynomials of degree six are used to construct a family of seven orthonormal polynomials of the same degree, named, as Bernstein orthonormal polynomials. The operational matrix of derivative was derived and applied for solving variationalproblems, by assuming representations of admissible functions by orthonormal Bernstein polynomials with coefficients to be determined, then the derived operational matrix of derivative was used for performing the derivative so that the variational problem reduces into quadratic programming problem. The aim of this technique has been to obtain effective algorithm that are suitable for the digital computers.

\section{References:}

1. Singh,A.K. and Singh V. 2009. The Bernstein Operational Matrix of Integration, Appl. Math. Scie. 13(49:.2427-2436.

2. Alipour M. and Rostomy D. 2011. Bernstein Polynomials for Solving Abel's Integral Equation, J. Math. and Computer Scie. 3(4) : 403-412.

3. Dixit S. and Singh V. 2010. Bernstein Direct Method for Variational Problems, Int. Math. Forum. 5 (48): 2351-2370.

4. Oian W. and Riedel M. 2011. Uniform Approximation and Bernstein Polynomials with Coefficients in the Unit Interval, Eur. J. Com. 32:448-463.

5. Ordokhani Y. and Far S.D. 2011. Application of the Bernstein Polynomials for Solving the Nonlinear FredholmIntegroDifferential Equations. J. Appl. Math. andBioin. 1 (2) : 13-31.

6. HosseiniS.Gh. 2011. A New Operationl Matrix of Derivative for Chebyshev Wavelets and Its Applications in Solving Ordinary Differential Equations with New Analytic Solution, App. Math. Sci. 5( 51): 2537-2548.

7. Doha E.H. and Bhrawy A.H. 2011. A Chebyshev Spectral Method Based on OperationallMatrix for 
Initial and Boundary Value Problems of Fractional Order, Comp. and Math. Appl. 62: 23642373.

8. MohammedAli. M.N. 2013. Approximate Solution to Calculus of Variational Problem Using Orthonormal Polynomials, J. Basic Edu. 19(77):17-23.

9. Maleki M. and Mashali M. 2010. A Numerical Solution of Problems in Calculus of Variation Using Direct Method and Non Classical Parameterization, J. of Comp.andappli. Math. 23:13641373.

10. Razzaghi M. and Yousefi. 1983. Legender Wavelets direct method for variational problems, J. Opt.1 Theory A ppl. 39(1), 143-149.

\section{مصفوفة العمليات الجديلة للمشتقة لمتعددات حدود برنشتن المتعامدة}

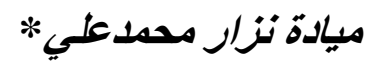

*قسم العلوم التطبيقية، الجامعة التكنولوجية

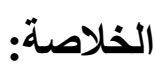

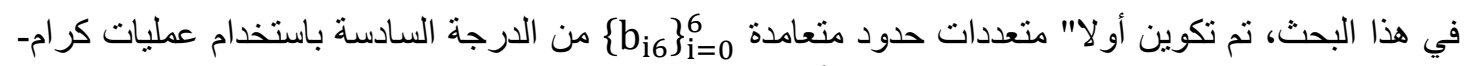

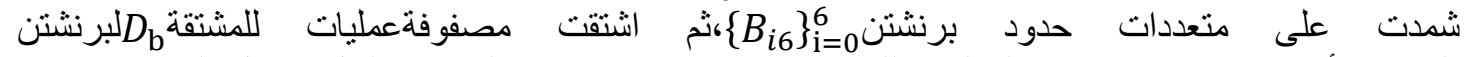
المتعامدة.أخير|"،طبقت مصفوفة العطليات للمشتئقة مع متعددات برنثنتن المتعامدة لحل مسائل التغاير تقريبيات باستخدام طريقة مباشرة. 